\title{
BOUNDS FOR CERTAIN SUMS; A REMARK ON A CONJECTURE OF MAHLER
}

\author{
BY \\ WOLFGANG M. SCHMIDT
}

1. Introduction. The main part of this paper consists in a proof of the following

THEOREM 1. Let

$$
Q(x, y)=Q_{n}(y) x^{n}+Q_{n-1}(y) x^{n-1}+\cdots+Q_{0}(y)
$$

be a polynomial in $x, y$ with integral coefficients. Write $m$ for the degree of $Q_{n}(y)$, $d$ for the total degree of $Q(x, y)$, and let $H \geqq 1, \rho>1 / 3$ be real numbers. Assume

$$
n \geqq 1,3 m \geqq n+3 ; \quad n \rho \geqq 1, m \rho \geqq 1,
$$

and assume

$$
Q(x, y)-k
$$

has no rational linear factor if $k \neq 0$. Then

$$
\sum_{|x| \leq H,|y| \leq H_{i} Q(x, y) \neq 0}|Q(x, y)|^{-\rho} \leqq \gamma_{d \rho} H^{2 / 8} .
$$

The sum is taken over integers $x, y$, and the constant $\gamma_{d_{\rho}}$ does not depend on the coefficients of $Q(x, y)$, except on the degree $d$.

REMARK. Examples of the type $Q(x, y)=\left(x^{2}-y\right) G(x, y)-1$ show that the exponent $2 / 3$ in (3) cannot be replaced by a constant less than $1 / 2$.

By $P=P(x)=a_{n} x^{n}+\cdots+a_{0}$, we denote polynomials in $x$ of degree $n$ and with integral coefficients. Define $H(P)$ by

$$
H(P)=\max \left(\left|a_{n}\right|, \cdots,\left|a_{0}\right|\right),
$$

and write $D(P)$ for the discriminant of $P$.

THEOREM 2. Let $\rho>1 / 3$ be real and assume

$$
n \geqq 3, \quad(n-1) \rho \geqq 1 .
$$

Then

$$
\sum_{P ; H(P) \leq H ; D(P) \neq 0}|D(P)|^{-\rho} \leqq \delta_{n \rho} H^{n-1 / 8} .
$$

Presented to the Society, January 26, 1961; received by the editors February 21, 1961. 
REMARKs. Only the bound $H^{n+1}$ is trivial; the bound $H^{n}$ was given in [3]. Our result is probably far from best possible. For $n=2$, the bound $H$ follows easily from the methods of [2](1).

In Mahler's classification of transcendental numbers $\zeta$, numbers $\theta_{n}(\zeta)$ $(n=1,2, \cdots)$ are defined as follows: $\theta_{n}(\zeta)$ is the least upper bound of the set of real numbers $\sigma$ such that there exists a sequence of polynomials $P_{1}(x), P_{2}(x), \cdots$ of degree $n$ and different from each other, satisfying

$$
\left|P_{i}(\zeta)\right|<H(P)^{-n \sigma} \quad(i=1,2, \cdots) .
$$

Mahler [6] conjectured $\theta_{n}(\zeta)=1$ almost everywhere. It is known that $\theta_{n}(\zeta) \geqq 1$ a.e. (see, for instance, $\left[7\right.$, p. 69]). $\theta_{1}(\zeta)=1$ a.e. follows from a theorem of Khintchine [4], $\theta_{2}(\zeta)=1$ a.e. was proved in $[5 ; 1 ; 2 ; 9], \theta_{3}(\zeta)=1$ a.e. has recently been proved by Volkmann $\left({ }^{2}\right)$. The best estimate so far for arbitrary $n$ was $\theta_{n}(\zeta) \leqq 2-2 / n$ a.e. $(n \geqq 2)$, and had been obtained by KaschVolkmann [3].

Theorem 3. Assume $n \geqq 3$ and suppose $\tau$ is a real number such that

Then

$$
\sum_{P ; H(P) \leq H ; D(P) \neq 0}|D(P)|^{-1 / 2}=O\left(H^{*}\right) .
$$

$$
\theta_{n}(\zeta) \leqq 1+\frac{\tau-2}{n} \text { almost everywhere. }
$$

Combining Theorems 2, 3 we obtain

THEOREM 4.

$$
\theta_{n}(\zeta) \leqq 2-7 / 3 n
$$$$
\text { a.e. }(n \geqq 3) \text {. }
$$

2. Two lemmas. We first give a short proof of the following result of Kasch-Volkmann [3].

LEMMA 1. To any $n \geqq 1$ and any $\rho>0$, there exists a constant $\gamma$ such that

$$
\sum_{|x| \leqq H_{i} P(x) \neq 0}|P(x)|^{-\rho} \leqq \begin{cases}\gamma H^{1-n} & \text { if } n \rho<1, \\ \gamma \log H & \text { if } n \rho=1, \\ \gamma & \text { if } n \rho>1\end{cases}
$$

for any $P$.

Proof. Write $P(x)=a_{n}\left(x-\alpha_{1}\right) \cdots\left(x-\alpha_{n}\right)$ and put $\alpha_{i}^{\prime}=R \alpha_{i}(i=1, \cdots, n)$ and

$$
\langle x\rangle=\min _{i=1, \ldots, n}\left|x-\alpha_{i}^{\prime}\right| .
$$

(1) Added in proof: H. Davenport in a paper to appear in Mathematika gives the bound $O\left(H^{2}\right)$ if $n=3, \rho=1 / 2$.

(2) According to a letter to the author. 
Since an inequality of the type $k \leqq\langle x\rangle\langle k+1$ has at most $2 n$ integral solutions $x$, since there are at most $2 H+1$ integers $x$ in $|x| \leqq H$, and since the function $g(k)=k^{-n p}$ is decreasing, we obtain

$$
\sum_{\left.|x| \leq B_{i}(x)\right\rangle_{2}}\langle x\rangle^{-n \rho} \leqq 2 n \sum_{k=1}^{2 H+1} k^{-n \rho} .
$$

There are at most $2 n$ integers $x$ having $\langle x\rangle\langle 1$, hence their contribution to the sum (7) is at most $2 n$. A bound for the remaining sum is furnished by $|P(x)|$ $\geqq\langle x\rangle^{n}$ and (8), whence the result.

Lemma 2. Assume the integers $n, m$ and the real $\rho>1 / 3$ satisfy (1). Define a sequence $\nu_{0}, \nu_{1}, \cdots$ by

$$
\begin{aligned}
& \nu_{0}=2 / 3-(4 m \rho)^{-1}, \\
& \nu_{i}=m n^{-1} \nu_{i-1}+(2 n \rho-1)(3 n \rho)^{-1} \quad(i=1,2, \cdots) .
\end{aligned}
$$

Then the elements of the sequence are positive and increasing. The sequence either tends to infinity or it has a finite limit larger than 1.

Proof. We have $\nu_{0}>0$ and

$$
\begin{aligned}
\nu_{1} & =m n^{-1}\left(2 / 3-(4 m \rho)^{-1}\right)+(2 n \rho-1)(3 n \rho)^{-1} \\
& =2 / 3+(2 m \rho-3 / 4-1)(3 n \rho)^{-1} \\
& >2 / 3>\nu_{0} .
\end{aligned}
$$

The sequence is increasing because $\nu_{j-1}<\nu_{j}$ together with (ii) implies $\nu_{j}<\nu_{j+1}$.

If $m \geqq n$, then the sequence tends to infinity and the lemma is true. Assume therefore $m<n$. Then there exists some $\nu$ such that

$$
m n^{-1} \nu+(2 n \rho-1)(3 n \rho)^{-1}=\nu .
$$

Clearly, $m n^{-1} K+(2 n \rho-1)(3 n \rho)^{-1} K<K$ if $\nu<K$. Using this property of $\nu$ as well as the fact that the sequence is increasing, we see that the sequence is bounded by $\nu$ and hence has a limit. Because of (9), the limit equals $\nu$. Solving (9) we find

$$
\nu=(2 n \rho-1)(3 n \rho-3 m \rho)^{-1},
$$

and $3 m>n+\rho^{-1}, 3 m \rho>n \rho+1,2 n \rho-1>3 n \rho-3 m \rho$ gives $\nu>1$.

Let $t$ be the smallest integer with $\nu_{t} \geqq 1$. Putting

$$
\mu_{i}=\nu_{i}-2 / 3 \quad(i=1,2, \cdots),
$$

we obtain

Write

$$
\begin{array}{ll}
0<\mu_{1}<\cdots<\mu_{t-1}<2 / 3 \leqq \mu_{t^{\prime}}, & \\
\mu_{i}=m n^{-1} \nu_{i-1}-(3 n \rho)^{-1} & (i=1,2, \cdots), \\
n \rho \mu_{i}-m \rho \nu_{i-1}=-1 / 3 & (i=1,2, \cdots) .
\end{array}
$$




$$
\begin{aligned}
Q_{n}(y) & =l\left(y-\beta_{1}\right) \cdots\left(y-\beta_{m}\right), \\
Q(x, y) & =Q_{n}(y)\left(x-\alpha_{1}(y)\right) \cdots\left(x-\alpha_{n}(y)\right) \quad \text { if } Q_{n}(y) \neq 0,
\end{aligned}
$$

and put $\beta_{i}^{\prime}=R \beta_{i}, \alpha_{j}^{\prime}(y)=R \alpha_{j}(y)$ and

$$
\begin{aligned}
\{y\} & =\min _{i=1, \ldots, m}\left|y-\beta_{i}^{\prime}\right|, \\
\{x \mid y\} & =\min _{j=1, \ldots, n}\left|x-\alpha_{j}^{\prime}(y)\right| .
\end{aligned}
$$

We split the sum (3) into $t+2$ parts $\sum_{00,} \sum_{0}, \sum_{1}, \cdots, \sum_{t}$, where $\sum_{00}$ consists of the terms of the sum where $\{y\}=0$, $\sum_{0}$ consists of the terms with $0<\{y\} \leqq H^{v 0}$, $\sum_{j}(j=1, \cdots, t-1)$ consists of the terms with $H^{v^{i-1}}<\{y\} \leqq H^{v i}$, $\sum_{t}$ consists of terms satisfying $H^{v_{t-1}}<\{y\}$.

Each of the sums $\sum_{j}(j=1, \cdots, t)$ will be split into three parts $\sum_{j 1}, \sum_{j 2}$ $\sum_{j 3}$ where the pairs $(x, y)$ involved satisfy

$$
\begin{aligned}
\sum_{j 1}: & & \{x \mid y\} & \geqq 1 \\
\sum_{j 2}: & H^{-\mu_{j}} \leqq & \{x \mid y\} & <1 \\
\sum_{j 3}: & & \{x \mid y\} & <H^{-\mu_{j}} .
\end{aligned}
$$

3. Bounds for $\sum_{00}, \sum_{0}, \sum_{j 1}, \sum_{j 2}$.

Lemma 3. $\sum_{00}=O\left(H^{2 / 8}\right)$.

More explicitly, $\sum_{00} \leqq \gamma H^{2 / 3}$, where $\gamma$ depends on $d$ and $\rho$ only. In all the equations of this section and the next, the $O$-symbol is to be understood in this way.

Proof. $\{y\}=0$ is equivalent with $Q_{n}(y)=0$. There are at most $m$ integers $y$ with $Q_{n}(y)=0$. For given $y_{0}$ having $Q_{n}\left(y_{0}\right)=0$, there are two alternatives. Either $Q_{n-1}\left(y_{0}\right)=\cdots=Q_{1}\left(y_{0}\right)=0$, or there exists some $h \geqq 1$ such that $Q_{h}\left(y_{0}\right) \neq 0$.

In the first case we have $Q_{0}\left(y_{0}\right)=0$ because otherwise the polynomial (2) with $k=Q_{0}\left(y_{0}\right) \neq 0$ would have the real linear factor $y-y_{0}$. Hence we have $Q\left(x, y_{0}\right) \equiv 0$ identically in $x$ and there is no contribution to the sum (3) with $y=y_{0}$.

In the second case $Q\left(x, y_{0}\right)$ is a polynomial in $x$ of some degree between 1 and $d$, and Lemma 1 yields 


$$
\sum_{|x| \leq B ; Q\left(x, y_{0}\right) \times 0}\left|Q\left(x, y_{0}\right)\right|^{-\infty}=O\left(H^{1-\rho}\right)=O\left(H^{2 / 8}\right) .
$$

LEMMA 4. $\sum_{0}=O\left(H^{2 / 8}\right)$.

Proof. For fixed $y_{0}$ with $\left\{y_{0}\right\}>0, Q\left(x, y_{0}\right)$ is a polynomial in $x$ of degree $n$, and $n \rho \geqq 1$ together with Lemma 1 gives the bound $O(\log H)$ for the sum over $x$. There are at most $\left(2 H^{0}+1\right) m$ integers $y_{0}$ with $0<\left\{y_{0}\right\} \leqq H^{0}$, and Lemma 4 follows from $H^{n} \log H=O\left(H^{2 / 8}\right)$.

Lemma 5. $\sum_{j 1}=O\left(H^{2 / 8}\right) \quad(j=1, \cdots, t)$.

Proof. We have $|Q(x, y)| \geqq\{y\}^{m}\{x \mid y\}^{n}$. Just as in (8), one can see that

$$
\sum_{|y| \leq H_{i}\{y\}_{21}}\{y\}^{-m \rho} \leqq 2 m \sum_{k=1}^{2 H+1} k^{-m p}=O(\log H)
$$

and, for fixed $y$,

$$
\sum_{|x| \leq B ;\{x|y| \geq 1}\{x \mid y\}^{-n \rho} \leqq 2 n \sum_{k=1}^{2 H+1} k^{-n \rho}=O(\log H) .
$$

The lemma follows.

Lemma 6. $\sum_{j 2}=O\left(H^{2 / 8}\right) \quad(j=1, \cdots, t)$.

Proof. This time we have $|Q(x, y)| \geqq\{y\}^{m}\{x \mid y\}^{n} \geqq H^{m y_{j-1}} H^{-n \mu_{j}}$. There are at most $2 n$ integers $x$ with $\{x \mid y\}<1$ for given $y$. Therefore

$$
\begin{aligned}
\sum_{j 2} & \leqq 2 n(2 H+1) H^{-m p r_{j}-1+n \rho m_{i}} \\
& =O\left(H^{2 / 8}\right)
\end{aligned}
$$

according to (11).

4. Bounds for $\sum_{j 3 .}$. The domain $H^{v_{j-1}}<\{\eta\} \leqq H^{\prime} i(j=1, \cdots, t-1)$ or the domain $H^{v t-1}<\{\eta\}$ consists of at most $2 m$ strips parallel to the $x$-axis. The intersection of these strips with $|\xi| \leqq H,|\eta| \leqq H$ consists of at most $2 m$ rectangles. The length of such a rectangle in the direction of the $x$-axis is $2 H$, the length in the direction of the $y$-axis at most $2 m H^{\prime \prime}(j=1, \cdots, t-1)$ or $2 m H$. From now on, we keep $j$ fixed, and $R$ will be a fixed rectangle of the type described above. We shall give bounds for the terms of $\sum_{j 8}$ where $(x, y) \in R$.

Write

$$
\alpha_{i j}(y)=\left(\alpha_{i}(y)+\alpha_{j}(y)\right) / 2 \quad(1 \leqq i, j \leqq n ; i \neq j),
$$

where $\alpha_{j}(y)$ is defined in (13). The elementary symmetric polynomials of the $C_{n, 2}$ functions $\alpha_{i j}(y)$ are polynomials in $y$ of degree $O(1)$, and therefore there exists a polynomial $R(x, y)$ of degree $C_{n, 2}$ in $x$ and of total degree $O(1)$ having 
$R\left(\alpha_{i j}(y), y\right)=0$ for $1 \leqq i, j \leqq n, i \neq j$ and $y$ arbitrary. Put $S(x, y)=Q(x, y) R(x, y)$. Writing $\alpha_{j}^{\prime}(y)$ for the real part of $\alpha_{j}(y)$ we find

$$
S\left(\alpha_{j}^{\prime}(y), y\right)=0 \quad(j=1, \cdots, n) .
$$

The real solutions of $S(\xi, \eta)=0$ will form certain curves in the plane. Their intersection with $R^{*}$ where $R^{*}$ is the rectangle containing $R$ in which the condition $|\xi| \leqq H$ is replaced by $|\xi| \leqq H+1$ will consist of a number of curves of the type

$$
x=x(y), \quad \phi \leqq y \leqq \psi,
$$

where $x^{\prime}(y), x^{\prime \prime}(y)$ exist and either

$$
x^{\prime \prime}(y) \geqq 0
$$

or

$$
x^{\prime \prime}(y) \leqq 0,
$$

and perhaps some line-segments of the type

$$
y=c, \quad-H-1 \leqq x \leqq H+1 .
$$

We denote the curves by $C_{1}, \cdots, C_{q}$ and have $q=O(1)$.

By $N\left(C_{l}\right)(l=1, \cdots, q)$ denote the set of integral pairs $(x, y) \in R$ such that for suitable $\zeta$

$$
(\zeta, y) \in C_{l}, \quad|x-\zeta|<H^{-\mu_{j}} .
$$

$\{x \mid y\}<H^{-\mu_{j}}$ implies $\left|x-\alpha_{k}^{\prime}(y)\right|<H^{-\mu_{j}}$ for some $k$ (q $\left.\leqq k \leqq n\right)$. Hence $(x, y) \in R$ together with $\{x \mid y\}<H^{-\mu_{i}}$ implies $(x, y) \in H\left(C_{l}\right)$ for some $l(1 \leqq l \leqq q)$, and we have

$$
\begin{aligned}
\sum_{j \boldsymbol{z}} & \leqq \sum_{l=1}^{q} \sum_{(x, y) \in N\left(C_{l}\right)}|Q(x, y)|^{-\rho} \\
& =\sum_{l=1}^{q} A\left(C_{l}\right) .
\end{aligned}
$$

Lemma 7. $A\left(C_{l}\right)=O\left(H^{2 / 3}\right)$ if $C_{l}$ is of the type (17).

Proof. For fixed $y=c$ with $\{c\}>0, Q(x, c)$ is a polynomial in $x$ of degree $n$, and $n \rho \geqq 1$ together with Lemma 1 gives the bound $O(\log H)$.

From now on we shall assume $C=C_{l}$ is of the type (16a). There are trivial changes in the argument if $C$ is of the type (16b). Construct the convex hull of $N(C)$ and in this convex hull consider the lattice-points $(x, y)$ such that $(x-\epsilon, y)$ is not in the hull if $\epsilon>0$. This set of lattice-points will be written $S(C)$, spine of $C . S(C)$ is not necessarily contained in $N(C)$.

LEMMA 8. The number of points of $N(C)$ which are not in $S(C)$ is $O\left(H^{2 / 8}\right)$. 


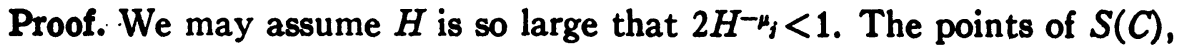
let us say $\left(x_{1}, y_{1}\right), \cdots,\left(x_{0}, y_{0}\right)$, can be ordered such that $y_{1}<y_{2}<\cdots<y_{0}$. Any $(x, y) \in N(C)$ has $y_{1} \leqq y \leqq y_{0}$. We shall prove that the number of points of $N(C)$ not in $S(C)$ with $y_{i} \leqq y \leqq y_{i+1}$ is at most

$$
2\left(y_{i+1}-y_{i}\right) H^{-\mu i} \text {. }
$$

Since

$$
\sum_{i=1}^{0-1}\left(y_{i+1}-y_{i}\right) \leqq 2 m H^{r^{i}}
$$

and since $\nu_{j}-\mu_{j}=2 / 3$ according to (10), the lemma follows.

Write $x=x_{i}(y)(i=1, \cdots, g-1)$ for the equation of the line through $\left(x_{i}, y_{i}\right)$ and $\left(x_{i+1}, y_{i+1}\right)$. Obviously, $x_{i}=x_{i}\left(y_{i}\right), x_{i+1}=x_{i}\left(y_{i+1}\right)$. We have $x>x_{i}(y)$ for every $(x, y) \in N(C)$ which is not in $S(C)$. As before, $x=x(y)$ is the equation of $C$. We find

$$
\begin{aligned}
& x_{i}>x\left(y_{i}\right)-H^{-\mu_{j}} \\
& (i=1, \cdots, g), \\
& x\left(y_{i}\right)-x_{i}\left(y_{i}\right)<H_{-} \mu_{i} \\
& (i=1, \cdots, g-1) \text {, }
\end{aligned}
$$

and similarly

$$
x\left(y_{i+1}\right)-x_{i}\left(y_{i+1}\right)<B^{-\mu_{j}} \quad(i=1, \cdots, g-1) .
$$

Using (19), (20) and $\left(x(y)-x_{i}(y)\right)^{\prime \prime} \geqq 0$ we find

$$
x(y)-x_{i}(y)<H^{-\mu_{i}} \text { if } y_{i} \leqq y \leqq y_{i+1} \quad(i=1, \cdots, g-1) .
$$

Any $(x, y) \in N(C)$ with $y_{i} \leqq y \leqq y_{i+1}$ has therefore

$$
x<x(y)+H^{-\mu_{j}}<x_{i}(y)+2 H^{-\mu_{j}} .
$$

Thus we have to show that there are at most $2\left(y_{i+1}-y_{i}\right) H^{-\mu_{i}}$ lattice-points in the parallelogram $y_{i} \leqq y \leqq y_{i+1}, x_{i}(y)<x<x_{i}(y)+2 H^{-\mu i}$, or at most $2 b H^{-\mu_{i}}$ lattice-points in the parallelogram

$$
0 \leqq y \leqq b, \quad \frac{a}{b} y<x<\frac{a}{b} y+2 H^{-\mu i},
$$

where $a=x_{i+1}-x_{i}, b=y_{i+1}-y_{i} . a$ and $b$ are relatively prime. Writing ( () for the difference between the smallest integer not smaller than $\zeta$ and $\zeta$ itself, (21) can be rewritten

$$
0 \leqq y \leqq b, \quad 0<\left(\frac{a}{b} y\right)<2 H^{-\mu_{j}} .
$$

But the number of integral solutions of (22) is equal to the largest integer not exceeding $2 b H^{-\mu_{i}}$. 
LEMMA 9. There are in $S(C)$ at most

$$
O\left(\min \left(r^{1 / 3} H^{2 / 3}, H\right)\right.
$$

points having $0<|Q(x, y)| \leqq r$.

Proof. We may assume $2 d r<H$, because otherwise $\min \left(r^{1 / 8} H^{2 / 8}, H\right)$ gives the trivial estimate $O(H)$. There are at most $d$ collinear points with $Q(x, y)$ $=k, k \neq 0$. Hence there are at most $2 d r$ collinear points having $0<|Q(x, y)|$ $\leqq r$.

Write $\left(x^{(1)}, y^{(1)}\right), \cdots,\left(x^{(p)}, y^{(p)}\right)$ for the points of $S(C)$ with $0<|Q(x, y)|$ $\leqq r$, and assume $y^{(1)}<\cdots<y^{(p)}$. Introduce the vectors

$$
v_{i}=\left(x^{(i+1)}-x^{(i)}, y^{(i+1)}-y^{(i)}\right) \quad(i=1, \cdots, p-1) .
$$

Since the points of $S(C)$ are on a convex polygon, it follows that $v_{i}=v_{i+k}$ implies $v_{i}=v_{i+1}=\cdots=v_{i+k}$ and that the points $\left(x^{(i)}, y^{(i)}\right), \cdots$, $\left(\left(x^{(i+k+1)}, y^{(i+k+1)}\right)\right.$ are collinear. Hence at most $2 d r$ of the vectors $v_{i}$ can be equal. We have

$$
\sum_{i=1}^{p-1}\left|v_{i}\right| \leqq 6 H+4,
$$

where $|v|$ denotes the length of $v$.

Order the set of all the nonzero vectors $w$ of $R^{2}$ with integral components in such a way that

$$
1=\left|w_{1}\right| \leqq\left|w_{2}\right| \leqq \cdots .
$$

Apparently $\left|w_{i}\right|>\gamma_{1} i^{1 / 2}\left(\gamma_{1}>0\right)$, and therefore

$$
\left|w_{1}\right|+\cdots+\left|w_{i}\right|>\gamma_{i} i^{i / 2} .
$$

If $p \leqq 2 d r$, then $p<(2 d r)^{1 / 8} H^{2 / 3}$ and the lemma is true. Hence we may assume $p>2 d r$. Write $p=2 d r s+u$, where $0 \leqq u<2 d r$.

$$
\begin{aligned}
\left|v_{1}\right|+\cdots+\left|v_{p}\right| & \geqq 2 d r\left(\left|w_{1}\right|+\cdots+\left|w_{s}\right|\right) \\
& \geqq 2 d r \gamma_{2} s^{3 / 2} \geqq \gamma_{z}(d) r q^{3 / 2} r^{-3 / 2} \\
& =\gamma_{3}(d) p^{3 / 2} r^{-1 / 2} .
\end{aligned}
$$

Using (23) we obtain $p^{3 / 2} r^{-1 / 2}=O(H), p=O\left(r^{1 / 3} H^{2 / 8}\right)$.

Lemma 10.

$$
\sum_{(x, y) \in S(C)}|Q(x, y)|^{-p}=O\left(H^{2 / 3}\right) .
$$

Proof. Write $a(r)$ for the number of points of $S(C)$ with $Q(x, y)=r$. We have to show that the (finite) sum

$$
\sum_{r=-\infty}^{\infty} a(r)|r|^{-p}=O\left(H^{2 / z}\right) .
$$


(The prime indicates that the term $r=0$ is omitted.) Using partial summation we find for $N>H$

$$
\begin{aligned}
\sum_{r=-N}^{N}{ }^{\prime} a(r)|r|-\rho & =\sum_{r=1}^{N}(a(r)+a(-r)) r-p \\
& =\sum_{r=1}^{N}\left(\sum_{k=-r}^{\dot{a}} a(k)\right)\left(r^{-\rho}-(r+1)^{-\rho}\right)+\sum_{k=-N}^{N} a(k)(N+1)^{-\rho} \\
& =O\left(\sum_{r=1}^{B} r^{1 / 3} H^{2 / 8} r^{-1-\rho}+\sum_{r=H+1}^{N} H r^{-1-\rho}+H N^{-\rho}\right) \\
& =O\left(H^{2 / 8}+H^{1-p}+H^{1-p}\right) \\
& =O\left(H^{2 / 8}\right) .
\end{aligned}
$$

Proof of Theorem 1. As explained in \$2, it is sufficient to give bounds for $\sum_{00}, \sum_{0,} \sum_{j 1}, \sum_{j g^{\prime}}, \sum_{j 8}(j=1, \cdots, t)$. Bounds for sums of the first four types are given in $\$ 3$. To estimate $\sum_{j 3}$, it is enough to estimate $A\left(C_{l}\right)$, as is seen by (18). This is done in Lemmas 7 through 10 .

REMARK. The crucial lemma of the proof is Lemma 10. Theorem 1 could be improved if this lemma could be improved.

5. Proof of Theorem 2. The discriminant $D(P)$ of a polynomial $P$ is a polynomial $D\left(a_{0}, a_{1}, \cdots, a_{n}\right)$ in the coefficients of $P$.

LEMMA 11.

(i) $D\left(a_{0}, \cdots, a_{n}\right)= \pm n^{n} a_{0}^{n-1} a_{n}^{n-1}+a_{0}^{n-2} R_{n-2}+\cdots+R_{0}$, where $R_{n-2}$, $\cdots, R_{0}$ are polynomials in $a_{1}, a_{2}, \cdots, a_{n}$.

(ii) The total degree of $D\left(a_{0}, \cdots, a_{n}\right)$ in $a_{0}$ and $a_{n}$ is $2 n-2$, and the only term of this degree is $\pm n^{n} a_{0}^{n-1} a_{n}^{n-1}$.

(iii) $D_{n}\left(a_{0}, \cdots, a_{n-1}, 0\right)= \pm a_{n-1}^{2} D_{n-1}\left(a_{0}, \cdots, a_{n-1}\right)$.

(iv) $D\left(a_{0}, \cdots, a_{n}\right)=D\left(a_{n}, \cdots, a_{0}\right)$.

Proof. Using $\pm a_{n} D(P)=R\left(P, P^{\prime}\right)$, where $R\left(P, P^{\prime}\right)$ is the resultant of $P$ and $P^{\prime}$, and the determinant representation of $R\left(P, P^{\prime}\right)$ (see, for instance, $[8, \S \S 29-31]$; observe that we write $P=a_{n} x^{n}+\cdots+a_{0}$ while van der Waerden writes $\left.P=a_{0} x^{n}+\cdots+a_{n}\right)$, we find

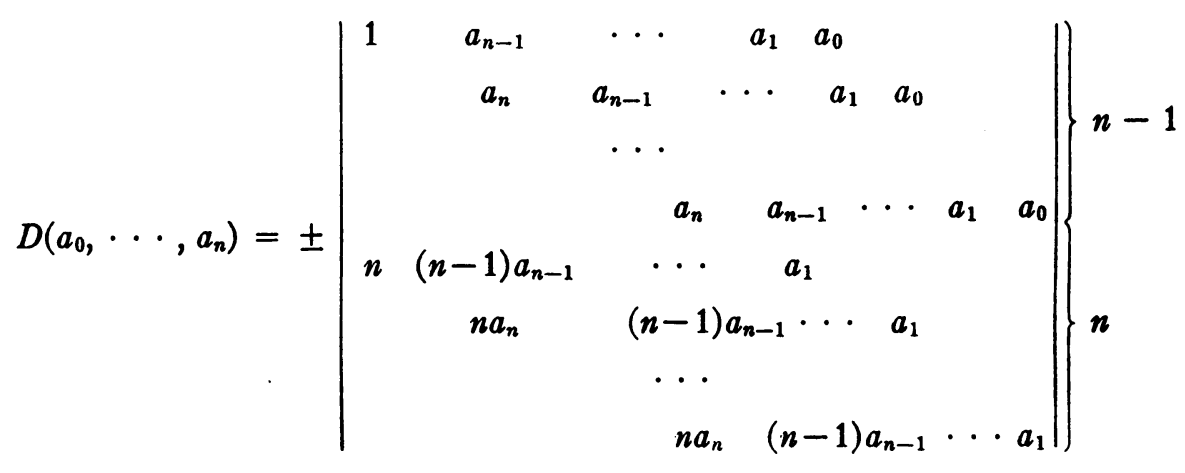


(i) and (ii) follow immediately from this representation while (iii) follows after a short computation using the determinant representation of $D_{n}\left(a_{0}, \cdots, a_{n}\right)$ is well as of $D_{n-1}\left(a_{0}, \cdots, a_{n-1}\right)$.

To prove (iv) we have to show that $D(P)=D(\bar{P})$ where $P=a_{n}\left(x-\alpha_{1}\right) \ldots$ $\left(x-\alpha_{n}\right)$ and $\bar{P}=a_{0}\left(x-\alpha_{1}^{-1}\right) \cdots\left(x-\alpha_{n}^{-1}\right)$. Now

$$
\begin{aligned}
D(\bar{P}) & =a_{0}^{n-1} \prod_{i \neq j}\left(\alpha_{i}^{-1}-\alpha_{j}^{-1}\right)=\prod_{i \neq j}\left(\alpha_{i}-\alpha_{j}\right) a_{0}^{n-1} \prod_{i=1}^{n} \alpha_{i}^{-(n-1)} \\
& =\prod_{i \neq j}\left(\alpha_{i}-\alpha_{j}\right) a_{0}^{n-1}\left(a_{n} / a_{0}\right)^{n-1}(-1)^{n(n-1)}=a_{n}^{n-1} \prod_{i \neq j}\left(\alpha_{i}-\alpha_{j}\right) \\
& =D(P) .
\end{aligned}
$$

LEMMA 12. Let $n \geqq 3$. Keep $a_{1}, \cdots, a_{n-1}$ fixed and write

$$
D(x, y)=D\left(x, a_{1}, \cdots, a_{n-1}, y\right) .
$$

Then

(a) $D(x, y)= \pm n^{n} x^{n-1} y^{n-1}+x^{n-2} Q_{n-2}(y)+\cdots+Q_{0}(y)$.

(b) The total degree of $D(x, y)$ is $2 n-2$ and only the term $\pm n^{n} x^{n-1} y^{n-1}$ has this degree.

(c) $D(x, y)-k$ has no linear factor if $k \neq 0$.

Proof. (a) and (b) follow from (i) and (ii) of the previous lemma. As for (c), assume there would be some $k \neq 0$ such that $D(x, y)-k$ had a linear factor. If $x=\alpha z+\beta, y=\gamma z+\delta$ were the parameter equation of this line, then we had

$$
f(z)=D(\alpha z+\beta, \gamma z+\delta) \equiv k \neq 0
$$

identically in 8 .

If $\alpha \gamma \neq 0$, then $f(z)$ is a polynomial of degree $2 n-2$ according to (b), a contradiction. Hence either $\alpha=0$ or $\gamma=0$, and because of (iv) we may assume $\gamma=0$. We have $D(z, \delta) \equiv k \neq 0$ identically in $z$, which is conceivable only with $\delta=0$, according to (a). Hence $D(z, 0) \equiv k \neq 0$. Using (iii) and applying (i) to $D_{n-1}$ we find

$$
\begin{aligned}
0 \neq k \equiv D(z, 0) & = \pm a_{n-1}^{2} D_{n-1}\left(z, a_{1}, \cdots, a_{n-1}\right) \\
& = \pm a_{n-1}^{2}\left( \pm(n-1)^{n-1} z^{n-2} a_{n-1}^{n-2}+S(z)\right),
\end{aligned}
$$

where $S(z)$ is of degree $\leqq n-3$. But $k \neq 0$ implies $a_{n-1} \neq 0$ and $D(z, 0)$ is therefore a polynomial of degree $n-2>0$, and we reach a contradiction.

Proof of Theorem 2. Lemma 12 enables us to apply Theorem 1 on $D(x, y)$ and we obtain

$$
\begin{aligned}
\sum_{P ; B(P) \leq H ; D(P) \neq 0}|D(P)|^{-\rho} & =\sum_{\left|a_{1}\right| \leq H} \cdots \sum_{\left|a_{n-1}\right| \leq H} \sum_{|x| \leq H,|y| \leq H ; D(x, y) \neq 0}|D(x, y)|^{-\rho} \\
& =O\left(B^{n-1 / 8}\right) .
\end{aligned}
$$




\section{The conjecture of Mahler.}

LEMMA 13. Suppose $\sigma$ is a number such that the sum

$$
\sum_{H=1}^{\infty}\left\{\sum_{P ; B(P)-H ; D(P) \neq 0} B^{-9-\infty \times}|D(P)|^{-1 / 2}\right\}
$$

is convergent. Then $\theta_{n}(\zeta) \leqq 1+\sigma$ almost everywhere.

Proof. This lemma follows from the argument on pages 448-449 of [3].

Proof of Theorem 3. Assume that (6) holds for some $\tau$ and put $\sigma=\sigma$. $=(\tau-2) n^{-1}+\epsilon$ for some $\epsilon>0$. Using partial summation we find

$$
\begin{aligned}
\sum_{H=1}^{N} & \left\{\sum_{P ; H(P)=H ; D(P) \times 0} H^{-2-\sigma n}|D(P)|^{-1 / 2}\right\} \\
= & O\left[\sum_{H=1}^{N}\left\{\sum_{P_{i} B(P) \leq H ; D(P) \neq 0} H^{-8-\sigma n}|D(P)|^{-1 / 2}\right\}\right. \\
& =O\left(\sum_{H=1}^{N} H^{-8-\sigma n+r}+N^{-2-\sigma n} \sum_{P_{;} H(P) \leq N ; D(P) \neq 0}|D(P)|^{-1 / 2}\right] \\
& =O(1) .
\end{aligned}
$$

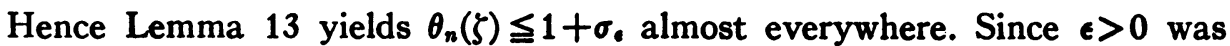
arbitrary, we obtain $\theta_{n}(\zeta) \leqq 1+(\tau-2) n^{-1}$ almost everywhere.

\section{REFERENCES}

1. J. W. S. Cassels, Some metrical theorems in Diophantine approximation. V, On a conjecture of Mahler, Proc. Cambridge Philos. Soc. vol. 47, (1951) pp. 18-21

2. F. Kasch, Über eine metrische Eigenschaft der S-Zahlen, Math. Z. vol. 70 (1958) pp. 263-270.

3. F. Kasch, and B. Volkmann, Zur Mahlerschen Vermutung uber S-Zahlen, Math. Ann. vol. 136, (1958) pp. 442-453.

4. A. Y. Khintchine, Einige Sutze uber Kettenbruche, mit Anwendungen auf die Theorie der Diophantischen Approximationen, Math. Ann. vol. 92 (1924) pp. 115-125.

5. J. F. Kubilyus, On the application of a method of Vinogradoff in a problem in metrical number-theory, (Russian), Dokl. Akad. Nauk SSSR (N.S.) vol. 67 (1949) pp. 783-786.

6. K. Mahler, Über das Mass der Menge aller S-Zahlen. Math. Ann. vol. 106 (1932) pp. 131-139.

7. T. Schneider, Einführung in die transzendenten Zahlen, Berlin-Grttingen-Heidelberg, Springer, 1957.

8. B. L. van der Waerden, Algebra, erster Teil, 4th ed., Berlin-Gottingen-Heidelberg, Springer, 1955.

9. B. Volkmann, Zur Mahlerschen Vermutung in Komplexen, Math. Ann. vol. 140 (1960) pp. 351-359.

UNIVERSITY OF Colorado,

Boulder, Colorado 